\title{
COSMOLOGY IN HORAVA-WITTEN M-THEORY
}

\author{
R. ARNOWITT, JAMES DENT AND B. DUTTA* \\ Center For Theoretical Physics, Department of Physics, Texas A\&M University, \\ College Station TX 77843-4242, USA \\ E-mail: arnowitt@physics.tamu.edu,j-dent@physics.tamu.edu, \\ duttabh@uregina.ca
}

\begin{abstract}
The cosmology of the Horava-Witten M-Theory reduced to five dimensions retaining the volume modulus is considered. Brane matter is considered as a perturbation on the vacuum solution, and the question of under what circumstances does the theory give rise to the standard RWF cosmology is examined. It is found that for static solutions, one obtains a consistent solution of the bulk field equations and the brane boundary conditions only for pure radiation on the branes. (A similar result holds if additional 5-branes are added in the bulk.) If one stabilizes the fifth dimension in an ad hoc manner, a similar inconsistency still occurs (at least for a Hubble constant that has no dependence on y, the fifth dimension.) Within this framework, the possibility of recovering the RWF cosmology still remains if the volume modulus and /or the distance between branes becomes time dependent, under which circumstances the Hubble constant must then depend on y (unless the fifth dimension and volume modulus expand at precisely the same rate).
\end{abstract}

The problem of obtaining a satisfactory cosmology in 5 dimensional (5D) theories so that the square of the Hubble constant $\mathrm{H}$ is proportional to the mass density has been the subject of much discussion since the initial work of Binetruy, Deffayet and Langlois ${ }^{1}$. Thus in the Randall-Sundrum 1 (RS) type model ${ }^{2}$ with two branes separated in the fifth dimension, it has been argued that it is insufficient to assume a static solution, and it is necessary to dynamically stabilize the fifth dimension to obtain the standard Robertson-Walker-Friedman (RWF) $\operatorname{cosmology}^{3}$ (see also talk by P.Kanti, these proceedings), though alternate views have been expressed ${ }^{4}$. The Horava-Witten (H-W) 11D theory ${ }^{5}$, compactified on a Calabi-Yau (CY) manifold becomes a 5D theory at low energy ${ }^{6}$. Here it has been argued in Refs. $^{7,8}$ that the correct RWF cosmology does arise (without stabilization) in the Hubble expansion era. However, in both these analyses, the boundary conditions on both end branes have not been imposed (and in Ref. ${ }^{8}$,

* present address: department of physics, university of regina, regina sk, s4s 0a2 canada 
the equations in the bulk have not been solved to check consistency). An approximate solution in the bulk satisfying the brane boundary conditions for the inflationary era was obtained in ${ }^{9}$, but this solution does not apply to Hubble expansion era where the string constants are much larger than the brane matter densities.

In this discussion we will consider the $\mathrm{H}-\mathrm{W}$ theory reduced to five dimensions for the simple case where we retain a volume moduli V. (Shape moduli will be neglected). We will solve the bulk equations for the static solution, impose fully all the boundary conditions on the two branes, and examine under what circumstances one can generate the conventional 4D RWF cosmology. We will see that the existence of moduli distinguishes the H-W M-theory from the phenomenological RS model.

\section{Horava-Witten M-Theory}

In this section we briefly summarize the Horava-Witten theory which is $11 D$ supergravity on an orbifold: $M_{10} \times X \times S^{1} / Z_{2}$ where $Z_{2}$ is the parity operation on the eleventh dimension. Yang Mills multiplets with gauge group $E_{8}$ exist on the orbifold planes at the fixed points $\left(x^{11}=0\right.$ and $\pi \rho)$ to cancel gravitational anomalies, and then cancellation of the $10 \mathrm{D}$ fermion anomaly produces a relation bewteen the Yang Mills gauge coupling constant and the $11 \mathrm{D}$ gravitational constant ${ }^{5}$. This resolves naturally the puzzle of conventional 10D heterotic string theory of why grand unification occurs at the GUT scale $M_{G} \simeq 3 \times 10^{16} \mathrm{GeV}$, rather than at the $4 \mathrm{D}$ Planck mass, for it is the $11 \mathrm{D}$ Planck constant that is fundamental, and its mass scale is $\sim M_{G}$ (while the orbifold length is $\sim 1 /\left(5 \times 10^{15} \mathrm{GeV}\right)$ i.e. about 10 times the compactifcation scale). If we retain only the Calabi-Yau volume modulus $V$, the reduced 5D theory Lagrangian takes the following simple form ${ }^{6}$ :

$$
\begin{aligned}
L & =-\frac{1}{2 k_{5}^{2}} \int_{M_{5}} \sqrt{-g}\left[R+\frac{1}{2} V^{-2} \partial_{\alpha} V \partial^{\alpha} V+\frac{3}{2} \alpha^{2}\right] \\
& +\frac{1}{k_{5}^{2}} \Sigma_{i} \int_{M_{4}^{i}} \sqrt{-g} V^{-1} 3 \alpha(-1)^{i+1} \alpha-\frac{1}{16 \pi \alpha_{G}} \Sigma_{i} \int_{M_{4}^{i}} \sqrt{-g} V t r F^{(i)}{ }_{\mu \nu}^{2} \\
& -\Sigma_{i} \int_{M_{4}^{i}} \sqrt{-g}\left[\left(D_{\mu} C\right)^{n}\left(D_{\mu} \bar{C}\right)^{n}+V^{-1} \frac{\partial W}{\partial C^{n}} \frac{\partial \bar{W}}{\partial \bar{C}^{n}}+D^{u} D^{u}\right]
\end{aligned}
$$

Note that the bulk cosmological constant and the brane cosmological constants are fixed by the same parameter $\alpha$ (which is scaled by the (integer) first Pontryagin class of the CY manifold). Thus unlike the RS model, there is no freedom to fine tune the cosmological constants. Numerically 
$\alpha=O\left(10^{15} \mathrm{GeV}\right)$ and so is much larger than the matter density (except in the inflationary era considered in ${ }^{9}$ where they are comparable).

We chose the following form for the 5D metric to describe he Hubble expansion era:

$$
d s^{2}=a^{2} d x^{k} d x^{k}-n^{2} d t^{2}+b^{2} d y^{2}
$$

where $y=x^{11}$ and $\mathrm{a}, \mathrm{n}$ and $\mathrm{b}$ are in general functions of $\mathrm{y}$ and $\mathrm{t}$. The relevant field equations are the Einstein equation arising from $G_{t t}, G_{k k}$, $G_{y y}, G_{t y}$ and the equation for $\mathrm{V}$, and are given in e. g. ${ }^{8}$. In addition there are the boundary conditions on the 3 branes at the orbifold planes $y_{1}=0$ and $y_{2}=\pi \rho$ :

$$
\begin{aligned}
(-1)^{i}\left(\frac{1}{b} \frac{a^{\prime}}{a}\right)_{y_{i}} & =\frac{1}{6 M^{3}} \rho_{i} \\
(-1)^{i}\left(\frac{1}{b} \frac{n^{\prime}}{n}\right)_{y_{i}} & =-\frac{1}{6 M^{3}}\left(2 \rho_{i}+3 p_{i}\right) \\
\left(\phi^{\prime}\right)_{y_{i}} & =\left[\left(3 b \alpha-\frac{b}{M^{3}} \rho_{i n r}\right) e^{-\phi}\right]_{y_{i}}
\end{aligned}
$$

where $M$ is the $5 \mathrm{D}$ Planck mass, $\rho_{i}$ and $p_{i}$ are the total matter density and pressure on the two 3 branes, $V=e^{\phi}$, and $\rho_{i(n r)}$ is the massive (nonrelativistic) matter. (Note that Ref. ${ }^{8}$ omits the effects of the $V$ factors in this analysis.)

\section{Solution Of The 5D Equations}

The cosmological constants in the bulk and on the banes are of GUT size, while the matter density coming from the branes are generally very small throughout the Hubble expansion era. Thus one can solve the field equations perturbatively, first neglecting the brane matter, and then including it in higher approximations. This should be a good approximation except in the very early universe during the inflation era where there $(\rho) \sim\left(M_{G}\right)^{4}$. To zero'th approximation then, we neglect all brane matter which leads to the static vacuum solution of Ref. ${ }^{6}: a(y)=n(y)=f^{1 / 2} ; b(y)=b_{0} f^{2}$; $V(y)=b_{0} f^{3}$, where $f(y)=c_{0}+\alpha|y|$. This solution solves all field equations and boundary conditions exactly, preserves Poincare invariance and breaks 4 of the 8 supersymmetries, which is appropriate for getting $N=1$ supergravity when one descends to four dimensions. Thus it represents an appropriate choice for a vacuum metric. The parameters $a_{0}, b_{0}, c_{0}$ are arbitrary due to the existence of flat directions in the potential (which perhaps would be determined by non-perturbative contributions). We next include 
the matter on the brane as a perturbation that generates the Hubble expansion and calculate it's effects to linear order in $\rho$. We consider first a solution where both the fifth dimension and the CY volume remains static, and only $a(y, t)$ has time dependence to accommodate Hubble expansion:

$$
\begin{gathered}
a(y, t)=f^{1 / 2}(1+\delta a(y, t)) ; n(y)=b_{0} f^{1 / 2}(1+\delta n(y)) \\
b(y)=b_{0} f^{2}(1+\delta b(y)) ; V(y)=b_{0} f^{3}(1+\delta V(y))
\end{gathered}
$$

The Hubble constant is then $H=\dot{a} / a \simeq \delta \dot{a}$, where " (dot, prime)" means " $(t, y)$ " derivative. Similarly one has $\delta \ddot{a}=\dot{H}+H^{2}$. The boundary conditions of Eq. (3) then reduce to ( $i=1,2$ for the two branes):

$$
\begin{aligned}
\left(\delta a^{\prime}+\frac{1}{2} \frac{\alpha}{f} \delta V\right)_{i} & =\frac{(-1)^{i}}{6 M^{3}}\left(\frac{\rho}{f}\right)_{i} \\
\left(\delta n^{\prime}+\frac{1}{2} \frac{\alpha}{f} \delta V\right)_{i} & =-\frac{(-1)^{i}}{6 M^{3}}\left(\frac{2 \rho+3 p}{f}\right)_{i} \\
\left(\delta V^{\prime}+3 \frac{\alpha}{f} \delta V\right)_{i} & =-\frac{(-1)^{i}}{M^{3}}\left(\frac{\rho_{n r}}{f}\right)_{i}
\end{aligned}
$$

For the static solution, one may chose a special frame where $\delta b=0$. At first sight then, it would appear that a satisfactory solution should be available: there are three independent variables, $\delta a, \delta n, \delta V$ obeying second order differential equations, and so there will be a total of six constants of integrations which in addition to $\mathrm{H}$ would give seven quantities to satisfy the six boundary conditions of Eqs. 5. However, the boundary conditions are two point conditions (rather than "initial" conditions), and as is well known such conditions need not give consistent solutions to differential equations (e.g. a free particle where we specify the initial and final velocity (rather than the initial position and initial velocity) generally has no consistent solution without "fine tuning" the velocity choice). Indeed, the equations from $G_{t t}, G_{k k}, G_{y y}$ and $V$ allow one to obtain a first integral involving precisely the quantities appearing in Eq. (5):

$$
\begin{aligned}
\left(\delta a^{\prime}+\frac{1}{2} \frac{\alpha}{f} \delta V\right)_{i} & =\frac{b_{0}^{2}}{\alpha}\left[\int_{f_{1}}^{f} d f f^{3} H^{2}+C_{1}\right] \\
\left(\delta n^{\prime}+\frac{1}{2} \frac{\alpha}{f} \delta V\right)_{i} & =\frac{b_{0}^{2}}{\alpha}\left[\int_{f_{1}}^{f} d f f^{3}\left[H^{2}+2 \dot{H}\right]+C_{2}\right] \\
\left(\delta V^{\prime}+3 \frac{\alpha}{f} \delta V\right)_{i} & =-3 \frac{b_{0}^{2}}{\alpha}\left[\int_{f_{1}}^{f} d f f^{3}\left[4 H^{2}+2 \dot{H}\right]+C_{3}\right]
\end{aligned}
$$

In Eqs.(6), we have left the y dependence of $H(y, t)$ arbitrary though the simplest choice (e.g. used in ${ }^{3}$ ) would be to assume $H$ to be independent of 
y. In spite of this we see that there are only three constants of integration to satisfy the six constraints of Eqs.(6), and so a solution will exist only under special situations.

The boundary conditions of the first two of Eqs.(5) then yield the following results:

$$
\begin{aligned}
\int_{f_{1}}^{f_{2}} f^{3} H^{2} & =\frac{1}{6} \lambda\left(\frac{\rho_{2}}{f_{2}}+\frac{\rho_{1}}{f_{1}}\right) ; C_{1}=-\frac{\lambda}{6} \frac{\rho_{i}}{f_{i}} \\
\int_{f_{1}}^{f_{2}} f^{3} \dot{H} & =\frac{1}{4} \lambda\left(\frac{\rho_{2}+p_{2}}{f_{2}}+\frac{\rho_{1}+p_{1}}{f_{1}}\right) ; C_{2}=-\frac{\lambda}{2} \frac{p_{i}}{f_{i}}
\end{aligned}
$$

where $\lambda=\alpha / b_{0}^{2} M^{3}$. One has

$$
\dot{\rho}=-3 H(\rho+p)
$$

as a consequence of the Bianchi identities for the static solution, or alternately from the $G_{t y}$ equation $^{8}$. If $H$ is independent of $y$, one may integrate Eq.(7) and obtain the conventional relation between $H^{2}$ and $\rho$ (with an explicit evaluation of the $4 \mathrm{D}$ Newtonian constant $G_{N}$ ). However, the third equation in Eq.(5) imposes an additional constraint yielding:

$$
\int_{f_{1}}^{f_{2}} f^{3}\left[4 H^{2}+2 \dot{H}\right]=\frac{1}{3} \lambda\left(\frac{\rho_{2(n r)}}{f_{2}}+\frac{\rho_{1(n r)}}{f_{1}}\right) ; C_{3}=\frac{1}{3} \lambda \frac{\rho_{i(n r)}}{f_{i}}
$$

Inserting Eqs.(7) into Eq.(9) then requires

$$
\frac{\rho_{2}-3 p_{2}}{f_{2}}+\frac{\rho_{1}-3 p_{1}}{f_{1}}=2\left(\frac{\rho_{2(n r)}}{f_{2}}+\frac{\rho_{1(n r)}}{f_{1}}\right)
$$

which requires $p_{i}=1 / 3 \rho_{i}$, and $\rho_{i(n r)}=0$. Thus a consistent solution is obtained only for pure radiation, and non-relativistic matter (or a cosmological constant) is not accommodated by a static solution in the HoravaWitten model. This is true independent of the y dependence of $H$

\section{Alternate Possibilities}

One may extend the results of Sec. 3 to include an arbitrary number of 5branes in the bulk ${ }^{10}$. One again finds (at least for a Hubble constant that is independent of $y$ ) that a consistent solution occurs only if pure radiation is on the orbifold branes. The RS model is a phenomenology, and so one may add additional fields into the bulk in an ad hoc manner. In this way the fifth dimension can be stabilized by including a potential for a radion field. The RS model then gives rise to a correct RWF cosmology for arbitrary matter. In contrast, the H-W M-theory is a reasonably well defined theory, 
and one is not free to add additional fields into the bulk at will. However, one might speculate on the possibility that some non-perturbative potential exists between the two orbifold 3-branes that stabilizes the fifth dimension. This would correspond to discarding the $G_{y y}$ field equation ${ }^{3}$. One may still solve the remaining field equations for the static case, and again one finds that for a $y$-independent Hubble constant, the equations are inconsistent (except for the radiation case). A linear y-dependence also is inconsistent. (Of course if by hand one also discards the $\mathrm{V}$ modulus equation as well, one can get consistent solutions as the theory is then reduced to the RS model.)

The remaining possibility then is to consider dynamic solutions where the volume modulus becomes time dependent. This possibility is not without some interest, as it would imply that the gauge coupling constant would be time dependent over cosmological time scales, for which some data exists ${ }^{11}$. In this circumstances, one may show that the Hubble constant then must have $\mathrm{y}$-dependence, the Hubble constant on the distant brane being different from the one on the physical brane (unless the fifth dimension expands at precisely the same rate as the volume modulus). This more complicated possibility is under investigation.

\section{Acknowledgement}

This work was supported in part by the National Science Foundation Grant PHY-0101015.

\section{References}

\section{References}

1. P. Binetruy, C. Deffayet and D. Langlois, Nucl. Phys. B565(2000)269.

2. L. Randall and R. Sundrum, Phys. Rev. Lett. 83(1999)3370.

3. C. Csaki, M. Graesser, L. Randall and J. Terning, Phys. Rev. D62(2000)045015.

4. J. Khoury and R-J. Zhang, Phys. Rev. Lett. 89(2002)061302.

5. P. Horava and E. Witten, Nucl. Phys. B460(1996)506; Nucl. Phys. B475(1996)94.

6. A. Lukas, B. Ovrut, K. Stelle and D. Waldram, Nucl. Phys. B552(1999)246.

7. U. Ellwanger, hep-th/0001126.

8. K. Enqvist, E. Keski-Vakkuri and S. Rasanen, Nucl. Phys. B614(2001)388.

9. A. Lukas, B. Ovrut and D. Waldram, Phys. Rev. D61(2000)023506.

10. E. Copeland, J. Gray, A. Lukas and D. Skinner, hep-th/0207281.

11. J. Webb, M. Murphy, V. Flambaum and S. Curran, astro-ph/0210531. 